made for the case of non-uniform spacing. Another generalization, in which the approximating function takes the form

$$
f(k)=a_{1} r_{1}^{k}+a_{2} r_{2}^{k}+\cdots+a_{N} r_{N}^{k}=\sum_{i=1}^{N} a_{i} r_{i}^{k}
$$

where the $r_{i}$ are completely arbitrary real numbers, can also be made. The $a_{i}$ can be determined by a modification of the method described in this paper, however the procedure is extremely cumbersome and very decidedly offers no advantage over the obvious Cramer's Rule solution.

Mathematics Department

Virginia Polytechnic Institute

Blacksburg, Virginia

1. F. B. Hildebrand, Introduction to Numerical Analysis, McGraw-Hill, New York, 1956.

\title{
A New Algorithm for Diagonalizing a Real Symmetric Matrix
}

\section{By C. Donald La Budde}

Abstract. The algorithm described in this paper is essentially a Jacobi-like procedure employing Householder and Jacobi orthogonal similarity transformations successively on a real symmetric matrix to obtain, in the limit, a diagonal matrix of eigenvalues. The columns of the product matrix of all the orthogonal transformations, taken in the proper order, form a complete orthonormal set of eigenvectors.

1. Introduction. In this paper we describe a Jacobi-like procedure for diagonalizing a real symmetric matrix by means of orthogonal similarity transformations. The earliest such procedure was proposed by Jacobi in 1847 which involved the use of plane rotations, but required a computer search for the largest off-diagonal element, in absolute value. Later, procedures were proposed in which the offdiagonal elements were annihilated in sequence. The latter method, known as the cyclic Jacobi method, was discussed by Forsythe and Henrici (1). They showed that convergence of this method would only take place if the angle of rotation lay in a closed interval properly contained in the open interval $\left(\left(-\frac{1}{2}\right) \pi,\left(+\frac{1}{2}\right) \pi\right)$. The method described here employs successive Householder and Jacobi orthogonal similarity transformations in a sequential fashion to obtain, as in Jacobi methods, in the limit, a diagonal matrix of eigenvalues. The columns of the product matrix of all the Householder and Jacobi transformations employed form a complete set of orthonormal eigenvectors. Throughout this paper we will confine ourselves to the consideration of real symmetric matrices.

2. General Description of the Algorithm; Definitions and Notations. The general procedure may be described as follows: beginning with an arbitrary sym-

Received May 13, 1963. Revised July 10, 1963. This work was sponsored by the National Aeronautics and Space Administration, Washington 25, D. C. 
metric matrix $A=\left(a_{j k}\right)$ of order $n$, we perform successive Householder and Jacobi orthogonal similarity transformations on $A$ so that in the limit we obtain a matrix, orthogonally similar to $A$, of the form (as a partitioned matrix)

$$
\left(\begin{array}{c:c}
e_{1} & 0 \\
\hdashline 0 & A_{1}^{-}
\end{array}\right)
$$

where $A_{1}$ is a symmetric matrix of order $n-1$ and $e_{1}$ is an eigenvalue of $A$. Then $A$ is reduced, and the same procedure is applied to $A_{1}$, etc. until a diagonal matrix of eigenvalues and a complete orthonormal set of eigenvectors is obtained. We need only describe the reduction of $A$ to a matrix of the kind (2.1) since all other parts of the reduction will be identical.

We now describe a general $i^{\text {th }}$ step in the iteration designed to reduce the matrix $A$ to one of type (2.1). One step consists of two orthogonal similarity transformations: first, a Householder transformation which annihilates $n-2$ off-diagonal elements in the first row of $A$; and second, a Jacobi plane rotation which annihilates the remaining off-diagonal element in the first row of $\mathrm{A}$. At the beginning of a typical step the first row of $A$ appears thus:

$$
(x, 0, x, x, \cdots, x, x) \text {. }
$$

After the Householder and before the Jacobi plane rotation the first row appears thus:

$$
(x, x, 0,0, \cdots, 0,0) \text {. }
$$

Then after the Jacobi plane rotation the first row again has the appearance of (2.2).

We now take the following definitions and notation: let $M$ be any real matrix or vector. Then we define

$$
\|M\|^{2}=\text { sum of the squares of all of the elements of } M \text {. }
$$

Let $A^{(i-1)}=\left(a_{j k}^{(i-1)}\right)$ denote the matrix $A$ and its elements before the $i^{\text {th }}$ step of the iteration. We may write $A^{(i)}$ in partitioned form:

$$
A^{(i)}=\left(\begin{array}{c:c}
a_{11}^{(i)} & S^{(i) t} \\
\hdashline \bar{S}^{(i)} & \bar{A}_{1}^{(\bar{i})^{-}}
\end{array}\right)
$$

where $A_{1}^{(i)}$ is a symmetric matrix of order $n-1$ and $S^{(i)}$ is a column vector of order $n-1$. Let $B^{(i)}=\left(b_{j k}^{(i)}\right)$ be the matrix $A$ and its elements during the $i^{\text {th }}$ step, after the Householder transformation and before the Jacobi plane rotation. $B^{(i)}$ may also be written in partitioned form:

$$
B^{(i)}=\left(\begin{array}{c:c}
b_{11}^{(i)} & V^{(i) t} \\
\hdashline V^{(i)} & B_{1}^{(i)}
\end{array}\right)
$$

where $B_{1}^{(i)}$ is a symmetric matrix of order $n-1$ and $V^{(i)}$ is a column vector of order $n-1$. Also, we let $I_{n}$ be the identity matrix of order $n$.

The Householder transformation $H^{(i)}$ used during the $i^{\text {th }}$ step may be written as follows:

$$
H^{(i)}=\left(\begin{array}{c:c}
1 & 0 \\
\hdashline 0 & 0 \\
\hdashline I_{n-1}-2 \bar{W}^{(i)} \bar{W}^{(i) i}
\end{array}\right) .
$$


where $W^{(i) t}$ is a row vector of order $n-1$ with elements $\left(w_{j}^{(i)}\right) j=2, \cdots, n$, and

$$
W^{(i) t} W^{(i)}=1 \text {. }
$$

The Jacobi plane rotation $J^{(i)}$ may be written as follows:

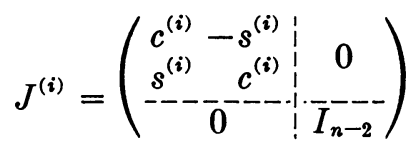

where $c^{(2)}, s^{(i)}$ are real numbers satisfying:

$$
c^{(i) 2}+s^{(i) 2}=1 \text {. }
$$

Finally we let $t^{(i)}=c^{(i)} / s^{(i)}$.

3. Proof of Convergence of the Algorithm. We first collect the following obvious results:

$$
\begin{gathered}
H^{(i)} A^{(i-1)} H^{(i)}=B^{(i)} ; \quad J^{(i) t} B^{(i)} J^{(i)}=A^{(i)} ; \\
V^{(i)}=\left(I_{n-1}-2 W^{(i)} W^{(i) t}\right) S^{(i-1)} ; \\
B_{1}^{(i)}=\left(I_{n-1}-2 W^{(i)} W^{(i) t}\right) A_{1}^{(i-1)}\left(I_{n-1}-2 W^{(i)} W^{(i) t}\right) ; \\
\left\|A^{(i-1)}\right\|=\left\|B^{(i)}\right\|=\left\|A^{(i)}\right\|=\|A\| ; \\
\left\|S^{(i-1)}\right\|=\left\|V^{(i)}\right\| ; \quad\left\|B_{1}^{(i)}\right\|=\left\|A_{1}^{(i-1)}\right\| ; \quad a_{11}^{(i-1)}=b_{11}^{(i)} .
\end{gathered}
$$

If we define $W^{(i)}$ (and hence $H^{(i)}$ ) as follows:

$$
\begin{array}{ll}
w_{2}^{(i)}=1 / \sqrt{2} & \\
w_{j}^{(i)}=a_{1 j}^{(i-1)} / \sqrt{2}\left\|S^{(i-1)}\right\| \quad j=3, \cdots, n
\end{array}
$$

then it is easily seen (3) that

$$
\begin{aligned}
\left|b_{12}^{(i)}\right| & =\left\|S^{(i-1)}\right\| \\
b_{1 j}^{(i)} & =0,
\end{aligned} \quad j=3, \cdots, n,
$$

and condition (2.8) holds. (At the beginning of a typical $i^{\text {th }}$ step $a_{12}^{(i-1)}=0$.) If we define $t^{(i)}$ (and hence $c^{(i)}, s^{(i)}$ up through relative signs) as follows:

$$
t^{(i)}=\left[a_{11}^{(i-1)}-b_{22}^{(i)}+\sqrt{\left(a_{11}^{(i-1)}-b_{22}^{(i)}\right)^{2}+4 b_{12}^{(i) 2}}\right] / 2 b_{12}^{(i)}
$$

from which follows:

$$
c^{(i) 2}=\left(\frac{1}{2}\right)\left[1+\left(a_{11}^{(i-1)}-b_{22}^{(i)}\right)\left[\left(a_{11}^{(i-1)}-b_{22}^{(i)}\right)^{2}+4 b_{12}^{(i) 2}\right]^{(-1 / 2)}\right],
$$

then it readily follows (2) that

$$
a_{12}^{(i)}=0 .
$$

Now we may state the principal theorem of this paper.

TheOREM: Let $H^{(i)}$ be defined by (3.2) and (3.3) and $J^{(i)}$ be defined by (3.6). Then

$$
\operatorname{Lim}_{i \rightarrow \infty} a_{11}^{(i)}=e_{1}
$$


an eigenvalue of $A$ and

$$
\operatorname{Lim}_{i \rightarrow \infty}\left\|S^{(i)}\right\|=0 .
$$

Hence the limiting form of $A$ will be of the type (2.1).

Proof: With $t^{(i)}$ defined by (3.6) the equation for $a_{11}^{(i)}$ is:

$$
a_{11}^{(i)}=a_{11}^{(i-1)}+\left(\frac{1}{2}\right)\left[b_{22}^{(i)}-a_{11}^{(i-1)}+\left[\left(a_{11}^{(i-1)}-b_{22}^{(i)}\right)^{2}+4 b_{12}^{(i) 2}\right]^{(1 / 2)}\right] .
$$

This shows that $a_{11}^{(i)} \geqq a_{11}^{(i-1)}$; we have strict inequality if $b_{12}^{(i)} \neq 0$. This shows that the $a_{11}^{(i)}$ form a monotonically increasing sequence of numbers bounded from above by $\|A\|$. This implies (3.9) which, together with (3.4) and (3.11), implies (3.10).

We note that condition (3.8) holds if the minus sign is chosen in front of the radical in the definition of $t^{(i)}(3.6)$. We may then state a corollary to the theorem.

CoRollary: If the minus sign in front of the radical in the definition of $t^{(i)}(3.6)$ is always chosen, then the conclusions of the theorem, (3.9) and (3.10), still follow.

Proof: The formula for $a_{11}^{(i)}(3.11)$ in this case will have a minus sign in front of the radical. This shows that the $a_{11}^{(i)}$ form a monotonically decreasing sequence of numbers bounded from below by $-\|A\|$. Hence the conclusions of the theorem readily follow.

It appears, at present, difficult to estimate the convergence rate of this algorithm. Some knowledge of the convergence rate may be obtained as follows. If we let

$$
U^{(i)}=S^{(i)} /\left\|S^{(i)}\right\|
$$

then it can easily be shown that:

$$
\left\|S^{(i)}\right\|^{2}=s^{(i) 2}\left[U^{(i-1) t} A_{1}{ }^{(i-1) 2} U^{(i-1)}-\left(U^{(i-1) t} A_{1}{ }^{(i-1)} U^{(i-1)}\right)^{2}\right] .
$$

The quantity inside the brackets may be estimated as follows:

$$
\begin{aligned}
|\bar{e}|^{2} \geqq U^{(i-1) t} A_{1}{ }^{(i-1) 2} U^{(i-1)}-\left(U^{(i-1) t} A_{1}{ }^{(i-1)} U^{(i-1)}\right)^{2} & \\
= & U^{(i-1) t} A_{1}{ }^{(i-1)}\left(I_{n-1}-U^{(i-1)} U^{(i-1) t}\right) A_{1}{ }^{(i-1)} U^{(i-1)} \geqq 0,
\end{aligned}
$$

where $\bar{e}$ is the eigenvalue of $A_{1}{ }^{(i-1)}$ with largest absolute value. The inequalities follow from the fact that $I_{n-1}-U^{(i-1)} U^{(i-1) t}$ is a projection onto the subspace of all $n-1^{\text {st }}$ order vectors orthogonal to $U^{(i-1)}$, along $U^{(i-1)}$. The first inequality of (3.14) will be an equality if $A_{1}{ }^{(i-1)}$ has two eigenvalues $\bar{e}$ and $-\bar{e}$ of maximum absolute value with corresponding normalized eigenvectors $X_{1}$ and $X_{2}$ and

$$
U^{(i-1)}=(1 / \sqrt{2}) X_{1}+(1 / \sqrt{2}) X_{2} .
$$

The last inequality of (3.14) will be an equality if and only if $U^{(i-1)}$ is an eigenvector of $A_{1}{ }^{(i-1)}$. If this is the case, then we have

$$
\sum_{k=2}^{n} a_{j k}^{(i-1)} a_{1 k}^{(i-1)}=\sum_{k=3}^{n} a_{j k}^{(i-1)} a_{1 k}^{(i-1)}=e a_{1 j}^{(i-1)} \quad j=2, \cdots, n,
$$

where $e$ is an eigenvalue of $A_{1}{ }^{(i-1)}$ corresponding to the eigenvector $U^{(i-1)}$. We note that $a_{12}^{(i-1)}=0$. In particular

$$
\sum_{k=3}^{n} a_{2 k}^{(i-1)} a_{1 k}^{(i-1)}=0 .
$$


But

$$
a_{1 j}^{(i)}=s^{(i)} b_{2 j}^{(i)} ; \quad a_{2 j}^{(i)}=c^{(i)} b_{2 j}^{(i)} ; \quad j=3, \cdots, n,
$$

so (3.17) becomes

$$
s^{(i-1)} c^{(i-1)} \sum_{j=3}^{n} b_{2 j}^{(i-1) 2}=0
$$

which implies

$$
\sum_{j=3}^{n} b_{2 j}^{(i-1) 2}=0
$$

if $s^{(i-1)}, c^{(i-1)}$ are both not zero. So if (3.14) approaches zero as $i$ approaches infinity, the algorithm causes the off-diagonal elements in both the first and second columns to approach zero. Thus, in this case the matrix $A$ will be reduced to a direct sum of a second order diagonal matrix and a matrix of order $n-2$. In general, it should be noted that although $\left\|S^{(i)}\right\|$ approaches zero as $i$ approaches infinity, the $\left\|S^{(i)}\right\|$ do not necessarily form a monotonically decreasing sequence.

Finally, we may make some remarks about the order in which the eigenvalues are computed. The algorithm appears, in most cases, to compute the eigenvalues in the order of algebraic magnitude, the algebraically largest being computed first, the next largest being computed second, etc., if $t^{(i)}$ is defined by (3.6). There certainly is no theorem to this effect, however; the most that can be said is the following. Let $e_{1}, e_{2}, \cdots, e_{n}$ be the eigenvalues of $A$ in the order in which they are computed, $e_{j} \neq e_{k}$ for $j \neq k$, and $b_{12}^{(i)} \neq 0$ for all $i$. Then the eigenvalue set $e_{j}$; $1 \leqq j \leqq n-1$ will not contain the smallest eigenvalue. The reason for this is the following. From (3.4), (3.6), (3.10) we can conclude

$$
\operatorname{Lim}_{i \rightarrow \infty} s^{(i)}=0 .
$$

From (3.7) and (3.21) we must have for sufficiently large $i$, and $b_{12}^{(i)} \neq 0$

$$
b_{22}^{(i)} \leqq a_{11}^{(i-1)}<a_{11}^{(i)}<e_{1} \text {. }
$$

The formula for $b_{22}^{(i)}$ is:

$$
b_{22}^{(i)}=U^{(i-1) t} A_{1}^{(i-1)} U^{(i-1)} .
$$

Because the eigenvalues of $A$ are distinct, the eigenvalues of $A_{1}{ }^{(i-1)}$ are also distinct and properly separate the eigenvalues of $A$. From this and (3.23) we can conclude:

$$
\text { (smallest eigenvalue of } \begin{aligned}
A) & <\left(\text { smallest eigenvalue of } A_{1}{ }^{(i-1)}\right) \\
& \leqq b_{22}^{(i)} \leqq\left(\text { largest eigenvalue of }{A_{1}}^{(i-1)}\right) \\
& <(\text { largest eigenvalue of } A) .
\end{aligned}
$$

The first inequality of (3.24) and (3.22) show that $e_{1}$ cannot be the smallest eigenvalue of $A$. A similar argument can be applied to $e_{2}, e_{3}$, etc. The reduction of the $n-1^{\text {st }}$ column and row places the larger of the two remaining eigenvalues in the $n-1^{\text {st }}$ row and column if $t^{(i)}$ is defined by (3.6). 
4. Conclusions. This method appears to be attractive for use in the problem of calculating all eigenvalues and a complete set of orthonormalized eigenvectors for the following reasons:

(a) Like the cyclic threshold Jacobi method, there is no search for largest pivotal elements.

(b) Unlike the cyclic threshold Jacobi method there is no limitation on the angle of rotation in the Jacobi rotation as Forsythe and Henrici (1) showed was necessary for convergence.

(c) Each iteration creates $n-1$ zeros at the cost of 3 square roots as compared to one zero in the Jacobi method at the cost of 2 square roots.

Acknowledgments. The author wishes to thank Dr. W. Givens, Dr. R. Gregory and Dr. R. C. Sahni for helpful discussions in connection with this work.

New York University

New York 3, New York

1. G. E. Forsythe \& P. Henrici, "The cyclic Jacobi method for computing the principal values of a complex matrix," Trans. Amer. Math. Soc. v. 94, 1960, p. 1-23.

2. H. H. Goldstine, F. M. Murray, \& J. Von NeumanN, "The Jacobi method for symmetric matrices," J. Assoc. Comput. Mach., v. 6, 1959, p. 59-96.

3. J. H. Wilkinson, "Householder's method for the solution of the algebraic eigenvalue problem," Comput. J. v. 3, 1960, p. 23-27.

\section{The Calculation of Certain Bessel Functions}

\section{By D. B. Hunter}

1. Introduction. The problem of calculating Bessel functions in a digital computer has engaged the attention of a number of authors in recent years, and a variety of methods is now available. Apart from the obvious use of the power-series for small arguments and the asymptotic expansions for large arguments, the methods which have been proposed include those based on the recurrence-relations (Stegun and Abramowitz [6]; Goldstein and Thaler [3]), phase amplitude methods (Goldstein and Thaler [2]), and methods based on quadrature formulas (Fettis [1], Luke [5]).

Particular difficulties arise in the case of the modified Bessel functions of the second kind, $K_{n}(z)$ with $z$ positive; for unless $z$ is small, the power-series for $K_{n}(z)$ resolves into a difference of two large numbers which are almost equal, with a consequent loss of significant digits. The asymptotic expansion and the phase-amplitude method, on the other hand, do not yield reasonable accuracy until $|z|$ is fairly large. Thus for medium-sized values some other approach must be used. For such values the quadrature methods are convenient.

2. Quadrature Methods. The methods of Fettis [1] and Luke [5] are based on the application of numerical integration to the expression

$$
K_{\nu}(z)=\int_{0}^{\infty} e^{-z \cosh \theta} \cosh \nu \theta d \theta
$$

Received August 13, 1962. Revised July 22, 1963. 